\title{
Axino dark matter in moduli-induced baryogenesis
}

\author{
Koji Ishiwata \\ Deutsches Elektronen-Synchrotron DESY, \\ 22607 Hamburg, Germany \\ E-mail: koji.ishiwata@desy.de
}

ABSTRACT: We consider axino dark matter in large $R$-parity violation (RPV). In modulidominated universe, axino is produced thermally or non-thermally via saxion decay, then late-decaying moduli dilute axino density, which results in the right abundance to explain the present dark matter. At the same time baryon asymmetry is generated due to moduliinduced baryogenesis via the large RPV. Axino is cosmologically stable in spite of the large $\mathrm{RPV}$ since its decay rate is suppressed by the axion decay constant, heavy squark mass or kinematics.

KeYwords: Supersymmetry Phenomenology

ARXIV EPRINT: 1407.1827 


\section{Contents}

1 Introduction 1

2 Cosmological scenario $\quad 2$

2.1 Moduli-dominated universe 2

2.2 Saxion decay 4

2.3 Axino production $\quad 6$

2.4 Axion production 9

$\begin{array}{lll}2.5 & \text { Axino stability } & 10\end{array}$

$\begin{array}{lll}3 & \text { Results } & 12\end{array}$

4 Conclusion $\quad 15$

\section{Introduction}

The discovery of the Higgs boson at the LHC confirmed the standard model of particle physics $[1,2]$. So far no phenomenon, which shows severe inconsistency with the standard model (SM), has been reported on the ground-based experiments (except for neutrino oscillation). In cosmology, however, it is clear that we need new physics beyond the standard model. First of all, the standard cosmology can not explain the existence of dark matter (DM). In addition, the baryon density predicted in the standard model is too small to account for the observed value. Supersymmetry (SUSY) is a promising solution to the issues. On top of that, string theory, which requires supersymmetry for the consistency, is a viable candidate for the theory of everything.

However, such an extension may cause another problem especially in cosmology. Moduli fields, which must be stabilized to compactify the extra dimensions in string theory, may be destabilized during inflation if the inflation scale is very high, which is indicated by the recent BICEP2 observation [3]. Even if the destabilization is avoided in some ways [4, 5], it is likely that moduli are displaced far from their true minima at the end of inflation. Then moduli start to oscillate, and soon dominate the energy density of the universe. The moduli-dominated universe ends when moduli decay, accompanying a huge entropy injection. This is potentially problematic because such substantial an entropy production dilutes pre-existing matter density, e.g., due to leptogenesis [6] as for baryon density. Then it is difficult to lead to big bang nucleosynthesis (BBN) and the structure formation of the universe. Possible ways out are following: production of a large amount of the matter density before moduli decay or generation of the matter density after moduli decay. As for baryonic matter, Affleck-Dine mechanism [7, 8] is a typical example of the former one. On 
the other hand, late-decaying gravitino [9] or saxion [10] can also produce baryon asymmetry, which corresponds to the latter. Recently another mechanism, moduli-induced baryogenesis, was proposed [11]. It was shown in ref. [11] that Kachru-Kallosh-Linde-Trivedi scenario [12] provides some ingredients needed for baryogenesis, such as large enough CP phase and suitable mass spectrum for superparticles, if at least two non-perturbative terms for a modulus are introduced in the superpotential. Then subsequent decays of gluino and squarks from moduli produce sufficient baryon asymmetry. In those baryogenesis due to late-decaying particle, however, a large $R$-parity violation (RPV) is assumed, which makes lightest superparticle (LSP) unstable. This is a downside to accounting for dark matter.

In this paper we consider axino LSP with large $R$-parity violation. Introducing the axion supermultiplet is motivated by Peccei-Quinn (PQ) mechanism [13, 14], which solves the strong $\mathrm{CP}$ problem. Assuming that the fermionic component of the axion multiplet, axino, is the LSP, axino is copiously produced by its radial component, saxion, decay and the scattering from thermal plasma. Axino can be cosmologically stable even if the RPV is $O(1)$ because its decay rate is suppressed by the axion decay constant, squark mass or kinematics. After saxion decay, moduli decay follows to dilute axino abundance, which results in the observed relic of dark matter. At the same time, baryon asymmetry of the universe is generated in moduli-induced baryogenesis with the RPV.

\section{Cosmological scenario}

In this section we describe the basic picture of our scenario. In the scenario moduli dominate the total energy of the universe after inflation. Axino is produced thermally or by non-thermal saxion decay in the epoch of moduli domination. Eventually moduli decay and dilute the axino abundance, which gives the right value to explain DM. Here baryon asymmetry is generated from moduli decay as well due to $R$-parity violated interaction. The saxion decay also generates axion. Although it is diluted by moduli decay, the produced axion may give a sizable contribution to radiation as dark radiation. Finally the stability of axino under the RPV is discussed. ${ }^{1}$ Masses of saxion and axino are model-dependent $[15,17-20]$. Thus we treat them as free parameters. It will be shown that wider parameter region in terms of axino, saxion and moduli masses is allowed for this scenario (in section 3).

\subsection{Moduli-dominated universe}

Let us begin with the thermal history after inflation. As we mentioned in the Introduction, the modulus field tends to be displaced from its true minimum due to the deformed potential during inflation or due to the initial condition. Then after inflation, it starts to oscillate around the true minimum when the Hubble parameter $H$ reduces to moduli mass $m_{X}$. Assuming $T_{R}$, the reheating temperature after inflation, is comparable to $T_{X \text {,osc }}$, the

\footnotetext{
${ }^{1}$ Axino DM in moduli- and saxion-dominated universe is considered with conserved $R$-parity in ref. [15]. They studied a case in which the reheating temperature after inflation is relatively low and moduli and saxion start to oscillate before inflation ends. Axino thermal production is not taken into account here. See also ref. [16] for the recent study of the cosmology of non-thermal dark matter, including axino dark matter, in detail.
} 
temperature when modulus begins to oscillate, the energy density of modulus field $X$ per entropy density freezes after the oscillation starts at a value of

$$
\frac{\rho_{X}(T)}{s(T)}=\frac{1}{8} T_{X, \mathrm{osc}}\left(\frac{\delta X_{\mathrm{ini}}}{M_{\mathrm{Pl}}}\right)^{2} \equiv\left[\frac{\rho_{X}}{s}\right]_{\mathrm{osc}} .
$$

Here $T$ is the cosmic temperature, $\rho_{X}$ is the energy density of modulus, $s(T)$ is the entropy density and $\delta X_{\text {ini }}$ is the initial amplitude of $X$ measured from its true minimum. Typically we expect $\delta X_{\text {ini }} \sim M_{\mathrm{Pl}}$ where $M_{\mathrm{Pl}} \simeq 2.4 \times 10^{18} \mathrm{GeV}$ is the reduced Planck mass. $T_{X \text {,osc }}$ is estimated from the equation $H \simeq m_{X}$ as

$$
T_{X, \mathrm{osc}}=\left[\frac{90}{\pi^{2} g_{*}\left(T_{X, \mathrm{osc}}\right)}\right]^{1 / 4} \sqrt{M_{\mathrm{Pl}} m_{X}} \simeq 6.9 \times 10^{13} \mathrm{GeV}\left(\frac{m_{X}}{10^{10} \mathrm{GeV}}\right)^{1 / 2} .
$$

Here $g_{*}(T)$ counts degree of freedom of relativistic particles in the thermal bath. Due to their huge energy density, moduli soon dominate the energy density of the universe. The temperature when moduli begin to dominate the universe is estimated from the relation $\rho_{X} \simeq \rho_{R}$ as

$$
T_{\text {dom }} \simeq \frac{1}{6} T_{X, \text { osc }}\left(\frac{\delta X_{\text {ini }}}{M_{\mathrm{Pl}}}\right)^{2},
$$

where we have used eq. (2.1). It is seen moduli dominate the total energy density soon after starting to oscillate.

Since the energy density of moduli redshifts as $\rho_{X} \propto a^{-3}$, it is given by

$$
\rho_{X}(T)=\left[\frac{\rho_{X}}{s}\right]_{\text {osc }} s(T),
$$

until moduli decay. As $\rho_{\text {tot }}$, the total energy density of the universe, is equal to $\rho_{X}$ during moduli domination, the Hubble parameter in moduli-dominated universe is given by

$$
H \simeq \sqrt{\frac{\rho_{\mathrm{tot}}}{3 M_{\mathrm{Pl}}^{2}}} \simeq \frac{\sqrt{T_{\mathrm{dom}} s(T)}}{2 M_{\mathrm{Pl}}} .
$$

The epoch of moduli domination terminates when moduli decay to particles in minimal supersymmetric standard model (MSSM), and the universe turns into radiation domination. The temperature at the beginning of this radiation-dominated universe is determined by $H \simeq \Gamma_{X}$ as

$$
T_{X}=\left[\frac{90}{\pi^{2} g_{*}\left(T_{X}\right)}\right]^{1 / 4} \sqrt{M_{\mathrm{Pl}} \Gamma_{X}} \simeq 9.8 \times 10^{4} \mathrm{GeV}\left(\frac{m_{X}}{10^{10} \mathrm{GeV}}\right)^{3 / 2} .
$$

Here we have used the decay rate of moduli, which is given by [21-24]

$$
\Gamma_{X} \simeq \frac{c_{X}}{4 \pi} \frac{m_{X}^{3}}{M_{\mathrm{Pl}}^{2}}
$$

where $c_{X}$ is $O(1)$ constant and here and hereafter we take it as unity. ${ }^{2}$ The moduli masses have to be larger than around $100 \mathrm{TeV}$ in order not to destroy BBN. Even if $m_{X} \gtrsim 100 \mathrm{TeV}$

\footnotetext{
${ }^{2}$ In the numerical analysis, we use the results given in ref. [11]. Here we note that axino can be generated by gravitino decay which is from moduli. We ignore this contribution by assuming that the partial decay width of moduli to gravitino pair is suppressed. Such a suppression can happen due to mixing between moduli and SUSY breaking field [22, 24].
} 
is satisfied, however, a huge entropy production due to moduli decay may strongly dilute primordial relics, such as baryon and DM. The effect is described by a dilution factor, which is given by a ratio of entropy density before and after the moduli decay,

$$
d_{X}=\frac{3}{4} T_{X}\left[\frac{\rho_{X}}{s}\right]_{\mathrm{osc}}^{-1}=6 \frac{T_{X}}{T_{X, \mathrm{osc}}}\left(\frac{M_{\mathrm{Pl}}}{\delta X_{\mathrm{ini}}}\right)^{2} \simeq 8.5 \times 10^{-9}\left(\frac{m_{X}}{10^{10} \mathrm{GeV}}\right)\left(\frac{M_{\mathrm{Pl}}}{\delta X_{\mathrm{ini}}}\right)^{2} .
$$

The dilution is important to reduce over-produced axino (and axion), which is discussed below.

\subsection{Saxion decay}

Saxion is the radial component field of the axion supermultiplet. The axion supermultiplet is determined as a flat direction of the scalar potential given by the PQ fields. Here the PQ fields have non-zero PQ charges and break PQ symmetry spontaneously. We define the axion supermultiplet as

$$
A=\frac{1}{\sqrt{2}}(\sigma+i a)+\sqrt{2} \theta \tilde{a}+F \text {-term }
$$

Here $\sigma, a$ and $\tilde{a}$ are saxion, axion and axino, respectively. For later calculation we define the axion decay constant as $f_{a}=\sqrt{2 \sum_{i} q_{i}^{2} v_{i}^{2}}$ where $q_{i}$ and $v_{i}=\left\langle\Phi_{i}\right\rangle$ are the PQ charge and the vacuum expectation value (VEV) of a PQ field $\Phi_{i}$, respectively. If the domain wall number $N_{\mathrm{DW}}$ is not unity, then $f_{a}$ should be $\sqrt{2 \sum_{i} q_{i}^{2} v_{i}^{2}} / N_{\mathrm{DW}}$.

Similar to moduli, saxion tends to have initial amplitude around its true minimum after inflation then it starts oscillation when $H \simeq m_{\sigma}$ ( $m_{\sigma}$ is saxion mass). Around this period, moduli begin to dominate the total energy. If saxion starts to oscillate before moduli domination, the temperature at the beginning of the oscillation is given by

$$
T_{\sigma, \mathrm{osc}} \simeq\left[\frac{90}{\pi^{2} g_{*}\left(T_{\sigma, \mathrm{osc}}\right)}\right]^{1 / 4} \sqrt{M_{\mathrm{Pl}} m_{\sigma}}
$$

and its energy density to entropy ratio is fixed at

$$
\left[\frac{\rho_{\sigma}}{s}\right]_{\mathrm{osc}}=\frac{1}{8} T_{\sigma, \mathrm{osc}}\left(\frac{\delta \sigma_{\mathrm{ini}}}{M_{\mathrm{Pl}}}\right)^{2} .
$$

Here $\delta \sigma_{\text {ini }}$ is the saxion initial amplitude, which is expected to be order of $f_{a}$ to $M_{\mathrm{Pl}}$. The value depends on the saxion potential (see, e.g., refs. [25-27]). ${ }^{3}$ On the other hand, saxion starts to oscillate after the universe is dominated by moduli when $T_{\sigma, \text { osc }}<T_{\text {dom }}$. In such a case, saxion energy density per entropy density has a fixed value

$$
\left[\frac{\rho_{\sigma}}{s}\right]_{\mathrm{osc}}=\frac{1}{8} T_{\mathrm{dom}}\left(\frac{\delta \sigma_{\mathrm{ini}}}{M_{\mathrm{Pl}}}\right)^{2}
$$

\footnotetext{
${ }^{3}$ For example, saxion may be trapped at a large initial amplitude due to thermal effect as $\delta \sigma_{\text {ini }} \sim \alpha_{s} M_{\mathrm{Pl}}[25]$ or $O\left(10^{2} f_{a}\right)$ [26] especially in a class of model where there exist more than one PQ fields. On the other hand, in a single PQ field model saxion can be trapped at the origin [27]. We do not consider such a case in this paper.
} 
In our scenario we consider $m_{X}$ is relatively larger than $m_{\sigma}$. Then the energy density of moduli is much larger than that of saxion during the period of moduli domination in either case.

After the coherent oscillation, saxion decays to lighter particles. The decay rate depends on axion model, i.e., Kim-Shifman-Vainshtein-Zakharov (KSVZ) (or hadronic axion) model [28, 29] or Dine-Fischler-Srednicki-Zhitnitsky (DFSZ) model [30, 31]. In both the KSVZ and the DFSZ models saxion couples to axino and axion via the kinetic term, which is given as [18]

$$
\mathcal{L}_{\sigma}=\left(1+\frac{2 \xi}{f_{a}} \sigma\right)\left[\frac{1}{2}\left(\partial_{\mu} \sigma\right)^{2}+\frac{1}{2}\left(\partial_{\mu} a\right)^{2}+\frac{1}{2} \overline{\tilde{a}} i \not \partial \tilde{a}\right]
$$

where $\xi=2 \sum_{i} q_{i}^{3} v_{i}^{2} / f_{a}^{2}$. Here we have used $\tilde{a}$ as a four component axino spinor. From this interaction, the partial decay widths for $\sigma \rightarrow a a$ and $\sigma \rightarrow \tilde{a} \tilde{a}$ are computed as

$$
\begin{aligned}
\Gamma(\sigma \rightarrow a a) & =\frac{\xi^{2}}{32 \pi} \frac{m_{\sigma}^{3}}{f_{a}^{2}} \\
\Gamma(\sigma \rightarrow \tilde{a} \tilde{a}) & =\frac{\xi^{2}}{4 \pi} \frac{m_{\sigma} m_{\tilde{a}}^{2}}{f_{a}^{2}}\left(1-4 \frac{m_{\tilde{a}}^{2}}{m_{\sigma}^{2}}\right)^{3 / 2}
\end{aligned}
$$

where $m_{\tilde{a}}$ is axino mass. In the KSVZ model, the process $\sigma \rightarrow a a$ overwhelms the other decay modes if $\xi \sim O(1)$. We take $\xi=1$ unless otherwise noted. Then the total decay rate is given by $\Gamma_{\sigma} \simeq \Gamma(\sigma \rightarrow a a) .{ }^{4}$ On the other hand, in the DFSZ model, saxion interacts with Higgs doublets in $F$-term potential. Then saxion can decay to the SM-like Higgs pair, whose partial decay width is

$$
\Gamma(\sigma \rightarrow h h)=\frac{k_{\sigma}}{4 \pi} \frac{\mu^{4}}{f_{a}^{2} m_{\sigma}}\left(1-4 \frac{m_{h}^{2}}{m_{\sigma}^{2}}\right)^{1 / 2}
$$

where $k_{\sigma}$ is $O(1)$ constant, which is taken to be unity in the later numerical evaluation, and $\mu$ is the $\mu$ parameter in the MSSM superpotential. ${ }^{5}$ This decay mode dominates the total decay rate if $\mu \gtrsim m_{\sigma}$. Saxion can also decays to sfermion pairs if kinematically allowed. The decay rate for the process, however, is suppressed by $\left\langle H_{u(d)}\right\rangle^{2} / \mu^{2}$ times Yukawa coupling constant squared. $\left(\left\langle H_{u(d)}\right\rangle\right.$ is the VEV of up (down)-type Higgs.) Thus we ignore it. For later calculation, we define the branching fraction for axino pair production as

$$
\operatorname{Br}(\sigma \rightarrow \tilde{a} \tilde{a})=\frac{\Gamma(\sigma \rightarrow \tilde{a} \tilde{a})}{\Gamma_{\sigma}}
$$

In the KSVZ model, the branching ratio is simply given by $\operatorname{Br}(\sigma \rightarrow \tilde{a} \tilde{a}) \simeq 8 m_{\tilde{a}}^{2} / m_{\sigma}^{2}$ in the limit $m_{\sigma} \gg m_{\tilde{a}}$. This is also true in the DFSZ model when $\mu \lesssim m_{\sigma}$.

\footnotetext{
${ }^{4}$ There exist the decay modes to gauge bosons. However, they are sub-dominant since they are suppressed by gauge coupling constant and the loop factor.

${ }^{5}$ Suppose there are two PQ fields, $\Phi_{1}$ and $\Phi_{2}$, and both of them get VEVs as $\left\langle\Phi_{1}\right\rangle=\left\langle\Phi_{2}\right\rangle$. If $\Phi_{1}$ couples to up- and down-type Higgses (denoted as $H_{u}$ and $H_{d}$, respectively) in superpotential, $\lambda \Phi_{1} H_{u} H_{d}$ $\left(\lambda\left(\Phi_{1}^{2} / M_{\mathrm{Pl}}\right) H_{u} H_{d}\right)$, then $\mu=\lambda\left\langle\Phi_{1}\right\rangle\left(\lambda\left\langle\Phi_{1}\right\rangle^{2} / M_{\mathrm{Pl}}\right)$ and $k_{\sigma}=1(2)$.
} 


\subsection{Axino production}

Axino is the fermionic component of the axion supermultiplet. Axino can be produced in several ways; thermal production, non-thermal saxion decay or the next-LSP (NLSP) decay. The production due to the NLSP decay is negligible because the NLSP mainly decays to the SM particles via RPV. The other two, i.e., saxion decay and thermal production, are potentially important. In terms of yield variable $Y_{\tilde{a}} \equiv n_{\tilde{a}} / s$ ( $n_{\tilde{a}}$ is the number density of axino), the resultant abundance of axino is expressed as,

$$
Y_{\tilde{a}}=Y_{\tilde{a}}^{\mathrm{DEC}}+Y_{\tilde{a}}^{\mathrm{TH}},
$$

where $Y_{\tilde{a}}^{\mathrm{DEC}}$ and $Y_{\tilde{a}}^{\mathrm{TH}}$ are contributions from saxion decay and thermal production, respectively.

$Y_{\tilde{a}}^{\mathrm{DEC}}$ is easily obtained. Using eqs. (2.11) and (2.12), the present axino density due to saxion decay is given by

$$
Y_{\tilde{a}}^{\mathrm{DEC}}=\frac{1}{4} d_{X} \frac{\max \left\{T_{\sigma, \mathrm{osc}}, T_{\mathrm{dom}}\right\}}{m_{\sigma}}\left(\frac{\delta \sigma_{\mathrm{ini}}}{M_{\mathrm{Pl}}}\right)^{2} \operatorname{Br}(\sigma \rightarrow \tilde{a} \tilde{a}) .
$$

Here we note that the produced axino is diluted due to the late-decaying moduli, which is taken into account by the dilution factor $d_{X}$. There is an entropy production due to saxion decay. However, it is much smaller than the entropy production from moduli. This is because saxion decays before moduli, the energy density of saxion is smaller than that of moduli and that the branching fraction to the MSSM-sector particles in saxion decay is typically suppressed. Therefore, the dilution due to saxion decay is negligible compared to moduli decay.

The thermal production, on the other hand, is highly model-dependent. It is described by Boltzmann equation,

$$
\dot{n}_{\tilde{a}}+3 H n_{\tilde{a}}=C_{\text {prd }} .
$$

Here a dot means derivative with respect to the cosmic time and $C_{\text {prd }}$ is axino production rate per unit volume, which depends on the axion model. The solution of the Boltzmann equation in radiation domination is given by (using $\dot{T}=-H T$ )

$$
Y_{\tilde{a}}^{\mathrm{TH}}=\int d T \frac{C_{\mathrm{prd}}}{s(T) H T} .
$$

In the KSVZ model, axino is mainly produced by thermal scattering or decay of the particles in thermal plasma via strong interaction. For example, the production rate due to scattering processes, such as $\tilde{q} g \rightarrow \tilde{a} q, \tilde{g} g \rightarrow \tilde{a} g$, is roughly estimated as $C_{\text {prd }} \sim \frac{\alpha_{s}^{3}}{f_{a}^{2}} n_{\text {MSSM }}^{2}$ at high temperature. ( $\alpha_{s}$ is strong coupling constant and $n_{\mathrm{MSSM}}$ is the number density of the MSSM particle.) Then from eq. (2.21) the yield variable of axino is estimated as

$$
Y_{\tilde{a}, \mathrm{KSVZ}}^{\mathrm{th}} \sim O\left(10^{-3}\right) \times \frac{\alpha_{s}^{3} M_{\mathrm{Pl}} T_{R}}{f_{a}^{2}} .
$$


It is seen that the axino production is the most active at the highest temperature of the universe, i.e., $T_{R} \cdot{ }^{6}$ Thus the axino abundance is almost determined by the production from thermal plasma before moduli dominates the total energy, which guarantees that we have used eq. (2.21). More precise computation of the axino production in radiation domination is done by refs. [32-36]. In our later numerical calculation we adopt the result given in ref. [34] and fit their result as

$$
Y_{\tilde{a}, \mathrm{KSVZ}}^{\mathrm{th}} \simeq \min \left\{Y_{\tilde{a}}^{\mathrm{eq}}, 4 \times 10^{-3} \alpha_{s}^{3} \log \left(0.1 / \alpha_{s}\right)\left(\frac{T_{R}}{10^{4} \mathrm{GeV}}\right)\left(\frac{10^{11} \mathrm{GeV}}{f_{a}}\right)^{2}\right\}
$$

where only QCD interaction is considered. ${ }^{7}$ It is seen that the estimate given in eq. (2.22) roughly agrees with the expression. $Y_{\tilde{a}}^{\mathrm{eq}}$ is the value when axion is thermalized, and typically $Y_{\tilde{a}}^{\mathrm{eq}} \simeq 1.8 \times 10^{-3}$ using $g_{*}=228.75$. The decoupling temperature $T_{D}$ can be estimated by equating the scattering rate for the production process with the Hubble parameter and it is obtained as

$$
T_{D}^{\mathrm{KSVZ}} \sim 10^{8} \mathrm{GeV}\left(\frac{f_{a}}{10^{11} \mathrm{GeV}}\right)^{2}\left(\frac{0.04}{\alpha_{s}}\right)^{3}
$$

which is consistent with ref. [37]. Then axino is thermalized when $T_{R} \gtrsim T_{D}^{\mathrm{KSVZ}}$. Since we assume $T_{R} \sim T_{X \text {,osc }}$, axino is thermalized in a wide range of the parameter space.

Thermal production of axino in the DFSZ model is different from the one in the KSVZ model. As it is mentioned in refs. [34, 38-40], the scattering process via strong interaction is suppressed in high temperature regime. Instead, the production due to axino interaction with Higgs and Higgsino or stop and top is effective. For example, the production rate for the processes, such as $\tilde{H} t \rightarrow \tilde{a} t, \tilde{t} \bar{t} \rightarrow \tilde{a} h$, is roughly $C_{\text {prd }} \sim \frac{\mu^{2}}{\pi f_{a}^{2} T^{2}} n_{\mathrm{MSSM}}^{2}$. Thus axino production occurs mainly in a lower temperature regime. From this fact axino is produced after moduli dominates the total energy. The solution given in eq. (2.21) can be used for the axino production, except for using eq. (2.5) for the Hubble parameter. As a result, the yield variable of axino during the epoch of moduli domination is roughly obtained as

$$
\left.Y_{\tilde{a}, \mathrm{DFSZ}}^{\mathrm{th}}\right|_{\mathrm{XD}} \sim O\left(10^{-4}\right) \times \frac{\mu^{2}}{f_{a}^{2}} \frac{M_{\mathrm{Pl}}}{\sqrt{T_{\mathrm{dom}} \mu}} .
$$

The contribution from decay gives the same order. Here we have assumed that $T_{R}>\mu$. It is seen that the resultant abundance is highly suppressed by $T_{\text {dom. }}$. In addition, it is diluted by the late moduli decay. Thus the contribution of the thermal production to axino abundance before moduli decay is negligible in a wide parameter range. ${ }^{8}$

\footnotetext{
${ }^{6}$ Saxion decay reheats radiation during moduli domination. If the reheating temperature exceeds squark or gluino mass, then axino is also produced at the time of saxion decay. The production is, however, negligible since the reheating temperature is much smaller than $T_{R}$ and that the production is suppressed by $T_{\text {dom }}$ (see also later discussion).

${ }^{7}$ The fitting formula is applicable where $T_{R} \gtrsim 10^{4} \mathrm{GeV}$ and gluino or squarks are thermalized.

${ }^{8}$ Axino thermal production occurs after saxion decay if the decay reheats radiation to a temperature larger than $\mu$. The production results in the same order of yield variable given in eq. (2.25). Therefore it is negligible for the same reason.
} 
Axino is also produced in the era of radiation domination after moduli decay. Here $T_{X}$ plays the role of $T_{R}$ in the above discussion. In the KSVZ model, however, the axino production is negligible because $T_{X}$ is smaller than gluino or squark mass in a wide parameter region, i.e., processes, such as $g g \rightarrow \tilde{a} \tilde{g}, q g \rightarrow \tilde{a} \tilde{q}$, are kinematically suppressed and gluino and squarks are not thermalized. ${ }^{9}$ On the other hand, in the DFSZ model, axino production may be substantial since the production is effective at low temperature. The processes without external stop, such as $\tilde{H} t \rightarrow \tilde{a} t$ or $\tilde{H} \rightarrow \tilde{a} h$, contribute to the production because the number density of stop is Boltzmann-suppressed. Here we assume $T_{X}>\mu$. Then the production due to the scattering leads to axino yield variable after moduli decay as

$$
\left.Y_{\tilde{a}, \mathrm{DFSZ}}^{\mathrm{th}}\right|_{\mathrm{RD}} \sim O\left(10^{-4}\right) \times \frac{M_{\mathrm{Pl}} \mu}{f_{a}^{2}} .
$$

The contribution from Higgsino decay has the same order as one from the scattering. The above result roughly agrees with more accurate numerical calculation in the literature. Using the recent result given in ref. [40], the yield variable of axino is read as ${ }^{10}$

$$
Y_{\tilde{a}, \mathrm{DFSZ}}^{\mathrm{th}} \simeq \min \left\{Y_{\tilde{a}}^{\mathrm{eq}}, 10^{-5}\left(\frac{\mu}{1 \mathrm{TeV}}\right)\left(\frac{10^{11} \mathrm{GeV}}{f_{a}}\right)^{2}\right\} .
$$

Here axino is thermalized when $T_{D}^{\mathrm{DFSZ}} \gtrsim \mu$, where decoupling temperature $T_{D}^{\mathrm{DFSZ}}$ is given by

$$
T_{D}^{\mathrm{DFSZ}} \sim M_{\mathrm{Pl}} \frac{\mu^{2}}{f_{a}^{2}}
$$

Then the condition for axino thermalization becomes $\mu \gtrsim 10^{4} \mathrm{GeV}\left(f_{a} / 10^{11} \mathrm{GeV}\right)^{2}$. This estimate is roughly consistent with eq. (2.27).

In summary, axino yield variable due to the thermal production is given by

$$
Y_{\tilde{a}}^{\mathrm{TH}}=\left\{\begin{array}{ll}
d_{X} Y_{\tilde{a}, \mathrm{KSVZ}}^{\mathrm{th}} & (\mathrm{KSVZ}) \\
Y_{\tilde{a}, \mathrm{DFSZ}}^{\mathrm{h}} & (\mathrm{DFSZ})
\end{array} .\right.
$$

Then the density parameter of axino at present time is obtained as

$$
\Omega_{\tilde{a}}=m_{\tilde{a}} Y_{\tilde{a}}\left(\rho_{c} / s\right)_{0}^{-1}=\Omega_{\tilde{a}}^{\mathrm{DEC}}+\Omega_{\tilde{a}}^{\mathrm{TH}},
$$

where $\left(\rho_{c} / s\right)_{0} \simeq 3.6 h^{2} \times 10^{-9} \mathrm{GeV}$ for $h \simeq 0.67$ [41]. Here we have split two contributions for later convenience. For example, in the KSVZ model, they are typically

$$
\begin{aligned}
\Omega_{\tilde{a}}^{\mathrm{DEC}} h^{2} & \simeq 0.43 \times\left(\frac{m_{\tilde{a}}}{20 \mathrm{GeV}}\right)^{3}\left(\frac{10^{6} \mathrm{GeV}}{m_{\sigma}}\right)^{3}\left(\frac{m_{X}}{10^{10} \mathrm{GeV}}\right)^{3 / 2}\left(\frac{\delta \sigma_{\mathrm{ini}}}{M_{\mathrm{Pl}}}\right)^{2}, \\
\Omega_{\tilde{a}}^{\mathrm{TH}} h^{2} & \simeq 0.084 \times\left(\frac{m_{\tilde{a}}}{20 \mathrm{GeV}}\right)\left(\frac{m_{X}}{10^{10} \mathrm{GeV}}\right)\left(\frac{M_{\mathrm{Pl}}}{\delta X_{\mathrm{ini}}}\right)^{2} .
\end{aligned}
$$

Here we have used $T_{\mathrm{dom}}$ and $Y_{\tilde{a}}^{\mathrm{eq}}$ in the estimation of $\Omega_{\tilde{a}}^{\mathrm{DEC}}$ and $\Omega_{\tilde{a}}^{\mathrm{TH}}$, respectively. The expression of $\Omega_{\tilde{a}}^{\mathrm{DEC}}$ can be applied in the DFSZ model when $\mu \lesssim m_{\sigma}$.

\footnotetext{
${ }^{9}$ This fact is crucial for moduli-induced baryogenesis. Otherwise produced baryon would be washed out. Axino can be produced via RPV interaction, such as $q q \rightarrow \tilde{a} q$. We have checked that this production is negligible in the parameter region that we are interested in.

${ }^{10}$ In ref. [40], the result is given for the case relativistic stop is in thermal bath and its mass is larger than $\mu$. In such a case the yield variable is proportional to $\frac{M_{\mathrm{P} 1} \mu^{2}}{f_{a}^{2} m_{\tilde{t}}}$.
} 


\subsection{Axion production}

We have seen that saxion mainly decays to axion pair. The produced axion is relativistic thus behaves as radiation, which is so-called dark radiation. The additional degree of freedom in radiation is described in terms of the effective number of neutrinos $N_{\text {eff }}=$ $N_{\text {eff }}^{\mathrm{SM}}+\Delta N_{\text {eff }}$. Here $N_{\text {eff }}^{\mathrm{SM}}=3.046$ [42] is the prediction in the standard model. The result by Planck satellite [41], combined with the measurements of the present Hubble parameter by Hubble Space Telescope [43], gives $N_{\text {eff }}=3.83 \pm 0.54$ at $95 \%$ C.L.. When the data from WMAP9 [44], the Atacama Cosmology Telescope [45] and the South Pole Telescope [46, 47] are included, the analysis gives $N_{\text {eff }}=3.62_{-0.48}^{+0.50}$ at $95 \%$ C.L. [41]. Though the current observations are consistent with the SM value, the central values are slightly deviated from the SM prediction. We will see below that $\Delta N_{\text {eff }}$ can be $O(1)$ in our scenario.

Referring to refs. [48, 49], $\Delta N_{\text {eff }}$ in our scenario is given by

$$
\Delta N_{\text {eff }}=3\left[\frac{\rho_{a}}{\rho_{\nu}}\right]_{\nu \text { decp }}=\frac{43}{7}\left(\frac{10.75}{g_{*}\left(T_{X}\right)}\right)^{1 / 3}\left[\frac{\rho_{a}}{\rho_{R}}\right]_{X \text { dec }} .
$$

Here $\rho_{\nu}$ and $\rho_{a}$ are the energy density of neutrinos and axion, respectively, and " $\nu$ decp" means the values at neutrino decoupling. $\left[\rho_{a} / \rho_{R}\right]_{X \text { dec }}$ is the ratio of the energy density of axion produced by saxion and radiation at the time of moduli decay. Using $\rho_{\sigma} \simeq \rho_{a}$ at the time of saxion decay, it is straightforward to get

$$
\left[\frac{\rho_{a}}{\rho_{R}}\right]_{X \mathrm{dec}}=\frac{4}{3} d_{X} \frac{\left[\rho_{\sigma} / s\right]_{\mathrm{osc}}}{T_{X}}\left(\frac{\Gamma_{X}}{\Gamma_{\sigma}}\right)^{2 / 3}
$$

Assuming $\Gamma_{\sigma} \simeq \Gamma(\sigma \rightarrow a a)$ and using eq. (2.12), $\Delta N_{\text {eff }}$ is estimates as

$$
\Delta N_{\mathrm{eff}} \simeq 0.028\left(\frac{10^{10} \mathrm{GeV}}{m_{X}}\right)^{2}\left(\frac{m_{\sigma}}{10^{6} \mathrm{GeV}}\right)^{2}\left(\frac{f_{a} / \xi}{10^{11} \mathrm{GeV}}\right)^{4 / 3}\left(\frac{\delta \sigma_{\mathrm{ini}}}{M_{\mathrm{Pl}}}\right)^{2} .
$$

In this paper we impose a conservative bound $\Delta N_{\text {eff }} \lesssim 1$ on our scenario.

Axion is also produced by coherent oscillation when the Hubble parameter becomes comparable to axion mass. If moduli decays before the axion coherent oscillation, the abundance of the axion due to the oscillation is the conventional value given in ref. [50], i.e., $\Omega_{a}^{\text {c.o. }} h^{2} \simeq 0.2 \theta_{a}^{2}\left(f_{a} / 10^{12} \mathrm{GeV}\right)^{1.19}$. Here $\theta_{a}$ is the initial misalignment angle of axion and it should be small in order for axion not to overclose the universe if $f_{a} \gtrsim 10^{12} \mathrm{GeV}$. The tuning of $\theta_{a}$ is possible when PQ symmetry is broken during or before inflation. However, there is a constraint from the isocurvature perturbation (see, e.g., ref. [51]). If the inflation scale is very high, the axion decay constant is strictly constrained without any fine-tuning of $\theta_{a}[52,53]$. Meanwhile, if PQ symmetry is broken after inflation, the misalignment angle should be replaced by $\pi / \sqrt{3}$. Then the tuning is impossible and $f_{a}$ is severely constrained. ${ }^{11}$ Therefore $f_{a}$ should be suppressed in either case if the result of the BICEP2 experiment is

\footnotetext{
${ }^{11}$ In this case, the domain wall number should be unity. Even if $N_{\mathrm{DW}}=1$, axion is also produced from axionic string and axionic domain wall, which gives stringent constraint for the the decay constant, i.e., $f_{a} \lesssim(2.0-3.8) \times 10^{10} \mathrm{GeV}[54]$. Recently, however, it is pointed out that the axion from the walls and strings are suppressed so that this constrained may be irrelevant [55].
} 
confirmed. ${ }^{12}$ Since it is claimed that the BICEP2 result needs further analysis (e.g., [56]) and that the axion abundance from the coherent oscillation is model-dependent as seen above, we simply assume that the axion energy density due to the coherent oscillation is sub-dominant. ${ }^{13}$ It is straightforward to take into account the axion abundance from the coherent oscillation and consider mixed axion and axino DM. ${ }^{14}$

\subsection{Axino stability}

In our model we consider RPV for moduli-induced baryogenesis. Through RPV interaction, axino decays to SM particles even if it is the LSP. The renormalizable RPV interaction in superpotential is

$$
W_{\not R_{p}}=\mu_{i} L_{i} H_{u}+\lambda_{i j k} Q_{i} L_{j} D_{k}^{c}+\lambda_{i j k}^{\prime} L_{i} L_{j} E_{k}^{c}+\lambda_{i j k}^{\prime \prime} U_{i}^{c} D_{j}^{c} D_{k}^{c},
$$

where $L_{i}, E_{i}^{c}, Q_{i}, U_{i}^{c}, D_{i}^{c}$ are chiral superfields of left-handed lepton doublet, righthanded charged lepton, left-handed quark doublet, right-handed up-type quark, righthanded down-type quark, respectively. $i, j, k$ are generation indices. In the present paper, we will take phenomenological approach to determine the order of each RPV couplings as follows. In our model baryon asymmetry is generated through the RPV interaction. Among the four types of interactions, $U^{c} D^{c} D^{c}$ type is the most effective for moduli-induced baryogenesis. ${ }^{15}$ For example, $\lambda_{332}^{\prime \prime}$ can be order of unity evading from the severe constraint from proton decay, and generate the observed baryon asymmetry [11]. The other lepton-number violating couplings are partly constrained phenomenologically or they can be forbidden by generalized lepton parities or discrete $R$ symmetry. (see, e.g., refs. [58, 59]). ${ }^{16}$ Based on the facts, we simply consider a case where at least one of $\lambda_{i j k}^{\prime \prime}$ is $O(1)$ and the others are irrelevant. $^{17}$

Axino lifetime is determined by the process $\tilde{a} \rightarrow u_{i} d_{j} d_{k}$. Relevant interaction for this process is dimension-four axino-quark-squark coupling:

$$
\mathcal{L}_{\tilde{a}-q-\tilde{q}}=g_{\mathrm{eff}}^{(L / R)} \tilde{q}_{L_{i} / R_{i}} \bar{q}_{i} P_{R / L} \gamma_{5} \tilde{a},
$$

where $P_{R / L}=\left(1 \pm \gamma_{5}\right) / 2$ and $\tilde{q}_{L_{i} / R_{i}}$ is left-/right-handed squark. Here quark and squarks are in the MSSM sector. In the KSVZ model, although axino has no interaction with quark and squark in the MSSM sector at tree level, the effective interaction is induced at loop

\footnotetext{
${ }^{12}$ In such a case axino from saxion decay may be suppressed due to the suppression of the initial amplitude (see also discussion in section 3).

${ }^{13}$ If moduli decay is suppressed due to some approximate symmetry and moduli decay after the coherent oscillation begins, the axion abundance can be diluted by moduli decay. Similar case is discussed in ref. [25].

${ }^{14}$ See ref. [16] for mixed axion-axino DM scenario.

${ }^{15}$ In ref. [57] a simple baryogenesis is suggested in a minimal extension of the standard model by using $u d d$ type higher dimension operator, which also contains a DM candidate.

${ }^{16}$ Nonrenormalizable operator which preserves lepton number, such as $Q Q Q H_{d} / \Lambda$, might exist. However, this is irrelevant in the discussion of axino stability since it is suppressed by some large scale $\Lambda$. We also refer to ref. [60] which gives phenomenological constraints on nonrenormalizable RPV operators for some interested readers.

${ }^{17}$ Axino DM with different RPV operators is studied in, e.g., refs. [61-64].
} 

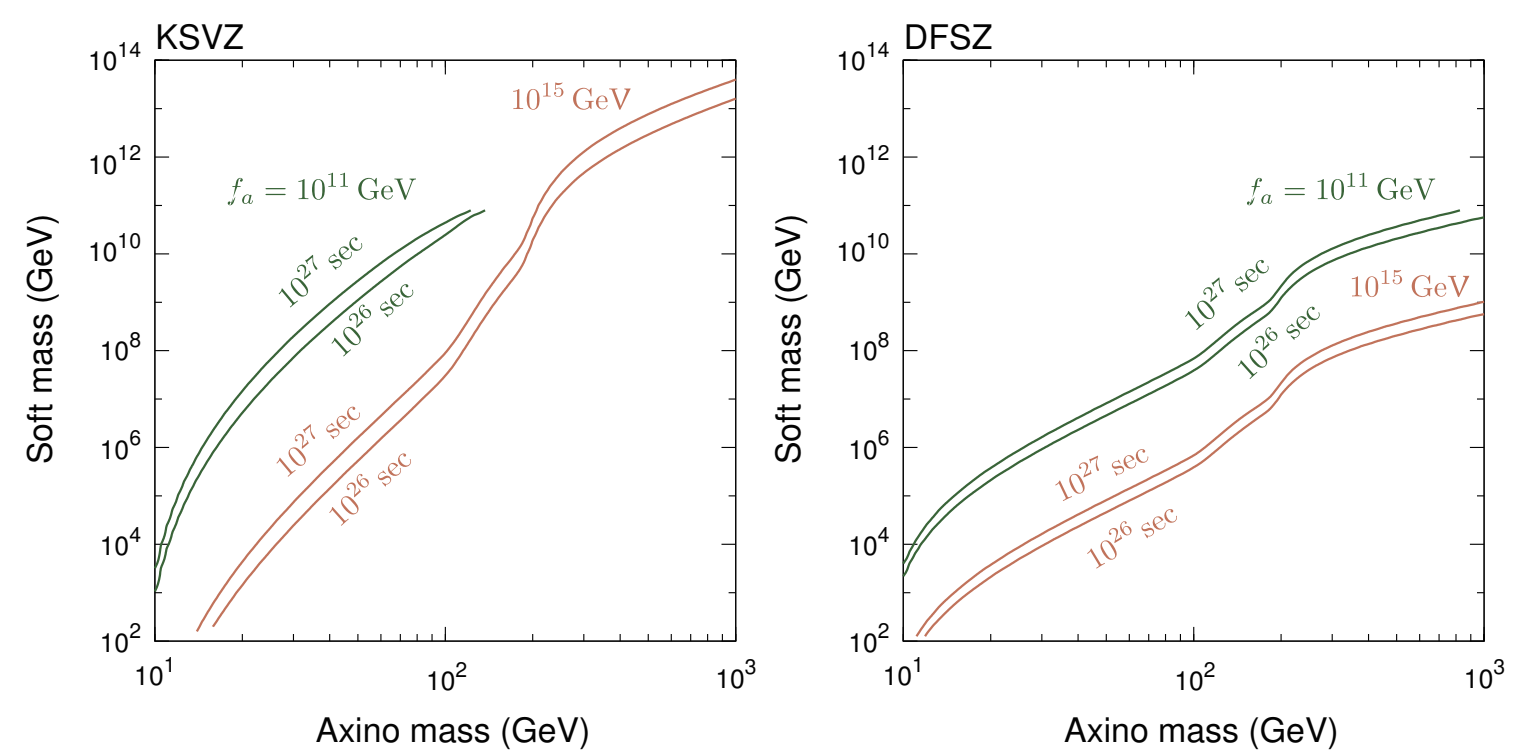

Figure 1. Contour of axino lifetime. Left and right panels correspond to the KSVZ and the DFSZ models, respectively. The contours of $\tau_{\tilde{a}}=10^{26}, 10^{27} \mathrm{sec}$ are depicted by taking $f_{a}=10^{11}$ (green), $10^{15} \mathrm{GeV}$ (red) in each panel. The plots are given in the region where the soft mass is less than $f_{a}$. Here we take $m_{\text {soft }}=m_{\tilde{g}}=m_{\tilde{t}}, \lambda_{332}^{\prime \prime}=1$ and the others are zero.

level. The effective coupling is given by [33]

$$
g_{\mathrm{eff}}^{(L / R)} \simeq \mp \frac{\alpha_{s}^{2}}{\sqrt{2} \pi^{2}} \frac{m_{\tilde{g}}}{f_{a}} \log \left(\frac{f_{a}}{m_{\tilde{g}}}\right),
$$

where $m_{\tilde{g}}$ is gluino mass. On the other hand, in the DFSZ model, tree-level interaction exists in $F$-term potential, given by [38]

$$
g_{\mathrm{eff}}^{(L / R)} \simeq \mp i \frac{m_{q}}{f_{a}}\left\{\begin{array}{l}
\cos ^{2} \beta \text { (for up-type quark) } \\
\sin ^{2} \beta \text { (for down-type quark) }
\end{array} .\right.
$$

Here $m_{q}$ is quark mass and $\tan \beta=\left\langle H_{u}\right\rangle /\left\langle H_{d}\right\rangle$. In our model the soft SUSY breaking scale tends to be large. To get the observed Higgs mass of around $126 \mathrm{GeV}[1,2], \tan \beta \simeq 1$ is required in the MSSM. Therefore we consider $\tan \beta=1$, which means that the axino decay is induced mainly by axino-top-stop interaction.

In the following discussion we assume that all superparticle (except Higgsino) in the MSSM sector have the same mass scale, which is characterized by the soft mass $m_{\text {soft }}$, i.e.,

$$
m_{\mathrm{soft}} \sim m_{\tilde{f}}, m_{\tilde{g}}, \text { etc. }
$$

where $m_{\tilde{f}}$ represents sfermion mass. In the calculation of axino lifetime, we use HELAS package [65].

Figure 1 shows contours of axino lifetime $\tau_{\tilde{a}}$. Here we take $m_{\text {soft }}=m_{\tilde{g}}=m_{\tilde{t}}\left(m_{\tilde{t}}\right.$ is stop mass), $\lambda_{332}^{\prime \prime}=1$ and the other $\lambda_{i j k}^{\prime \prime}$ are zero. ${ }^{18}$ Via $\lambda_{332}^{\prime \prime}$ axino decays to $t b s$ if kinematically

\footnotetext{
${ }^{18}$ In the computation we ignored left-right mixing in squark sector for simplicity. (Taking into account it is straightforward.) Here sbottom mediated diagram is neglected for simplicity by assuming stop is the lightest squark.
} 
allowed. If axino is lighter than top but heavier than $W$ boson, the final state is $W b b s$. The final state becomes five body in which off-shell $W$ boson decays when $m_{\tilde{a}} \lesssim m_{W}$. (In the five-body final state, we ignored fermion masses except for bottom quark.) Those behavior can be seen in the plot. When axino mass is around $W$ boson mass and top mass, the lifetime is enhanced. Then a large soft mass is required to suppress the lifetime. In the KSVZ model the lifetime is not strongly suppressed by the soft mass compared to in the DFSZ model. This is due to a factor of gluino mass in the effective $\tilde{a}-\tilde{q}-q$ coupling. Then the lifetime should be suppressed by even larger soft mass.

\section{Results}

Now we are ready to give numerical results. Before showing the results, we summarize the conditions which need to be satisfied for our scenario:

$$
\begin{aligned}
& \text { i) } T_{X} \gtrsim 10 \mathrm{MeV} \text {, } \\
& \text { ii) } \tau_{\tilde{a}} \gtrsim 10^{26} \mathrm{sec} \text {, } \\
& \text { iii) } \Gamma_{\sigma}>\Gamma_{X} \text {. }
\end{aligned}
$$

i) and ii) are the phenomenological constraints, i.e., moduli decays before BBN and axino should not produce any exotic cosmic rays. The last one is the condition in order for our scenario to work, i.e., saxion decays before moduli. In the KSVZ model, it is simply given by $m_{X} / m_{\sigma} \leq 4.2 \times 10^{4}\left(\frac{10^{11} \mathrm{GeV}}{f_{a} / \xi}\right)^{2 / 3}$ in $m_{\sigma} \gg m_{\tilde{a}}$ limit. It should be also reminded that we are interested in the mass spectrum, such as

$$
m_{\tilde{a}}<\left(\mu, m_{\sigma}, m_{\mathrm{soft}}\right)<m_{X} .
$$

In figure 2 , contours of $\Omega_{\tilde{a}}^{\mathrm{DEC}}=\Omega_{\mathrm{DM}}$ and $\Omega_{\tilde{a}}^{\mathrm{TH}}=\Omega_{\mathrm{DM}}\left(\Omega_{\mathrm{DM}} h^{2}=0.1196 \pm 0.0031\right.$ at $68 \%$ C.L. [41]) are plotted on $\left(m_{\sigma} / m_{\tilde{a}}, m_{X} / m_{\sigma}\right)$ plane. Here we take $m_{\tilde{a}}=20 \mathrm{GeV}$, $m_{\text {soft }}=m_{X} / 50, f_{a}=10^{11} \mathrm{GeV}, \delta X_{\text {ini }}=\delta \sigma_{\text {ini }}=M_{\mathrm{Pl}}$. Left (right) panel shows the result in the KSVZ (DFSZ) model. In the plot of the DFSZ model, we take $\mu=10^{2} m_{\tilde{a}}$. For the determination of the axino lifetime we take $\lambda_{332}^{\prime \prime}=\left(3 \times 10^{6} \mathrm{GeV} / m_{X}\right)^{1 / 4} / 2$ to explain the present baryon density [11] and the others are taken to be zero. $m_{X} \gtrsim 3 \times 10^{6} \mathrm{GeV}$ should be satisfied for the baryogenesis, which is also shown in dot-dashed line. In the figure shaded regions are excluded. Since axino mass is lighter than $W$ boson mass, the axino decay is five body. We found that the constraint $\tau_{\tilde{a}} \gtrsim 10^{26} \mathrm{sec}$ is much more stringent than the BBN constraint, which excludes the lower mass range. The bound is stronger in the KSVZ model due to the enhancement of the effective $\tilde{a}-\tilde{q}-q$ coupling. However, it turns out that the region $\Omega_{\tilde{a}} \simeq \Omega_{\mathrm{DM}}$ exists in the valid parameter region for both models. Two contributions, $\Omega_{\tilde{a}}^{\mathrm{DEC}}$ and $\Omega_{\tilde{a}}^{\mathrm{TH}}$, have different dependence on the mass parameters. In both models $\Omega_{\tilde{a}}^{\mathrm{DEC}}$ is the same and well agree with eq. (2.31). ${ }^{19}$ As for the thermal production, on the other hand, axino is thermalized in the region near $\Omega_{\tilde{a}}^{\mathrm{TH}} \simeq \Omega_{\mathrm{DM}}$ but diluted effectively to give the right amount in the KSVZ model, which is consistent with

\footnotetext{
${ }^{19}$ Except for low $m_{\sigma}$ range because $\sigma$ decay to Higgs pair changes $\operatorname{Br}(\sigma \rightarrow \tilde{a} \tilde{a})$.
} 

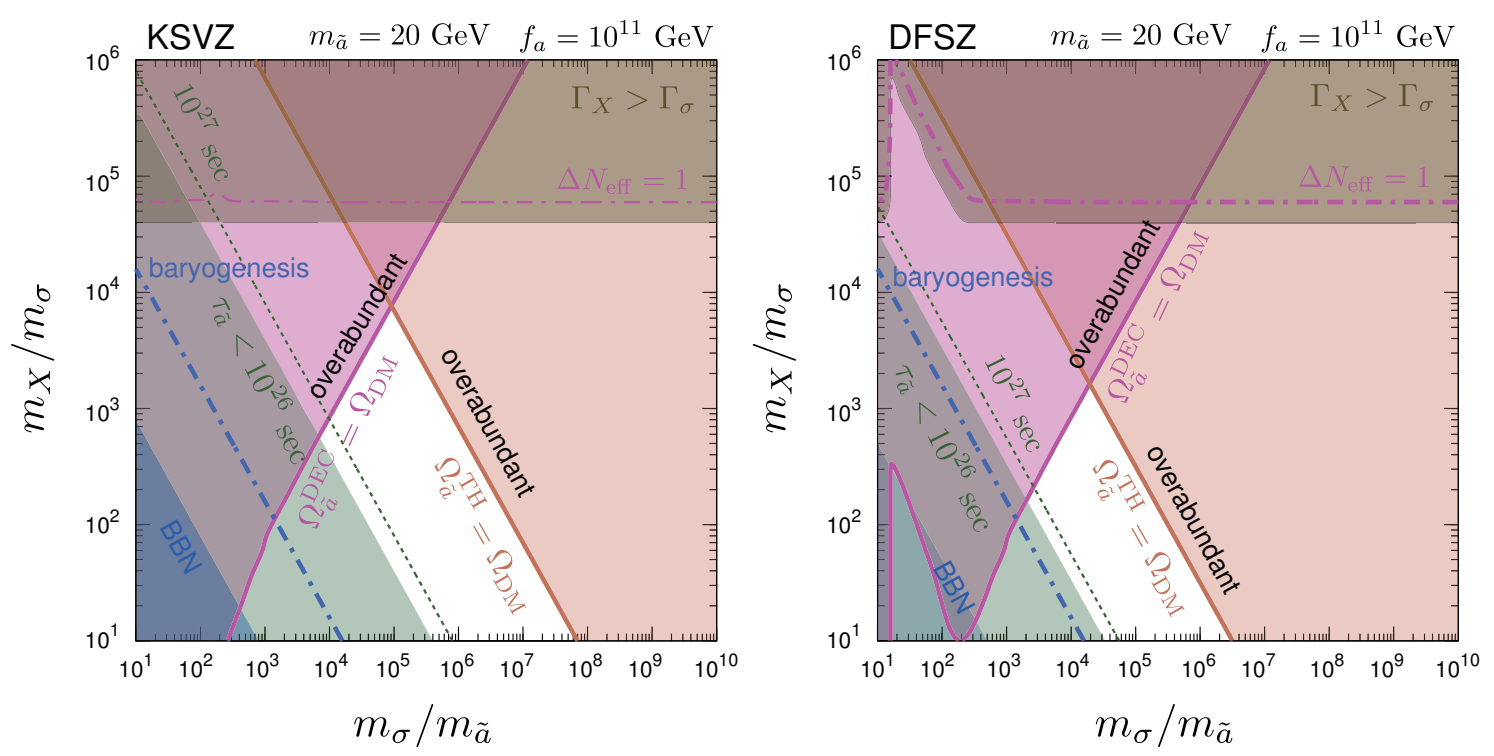

Figure 2. Contours of $\Omega_{\tilde{a}}^{\mathrm{DEC}}=\Omega_{\mathrm{DM}}$ and $\Omega_{\tilde{a}}^{\mathrm{TH}}=\Omega_{\mathrm{DM}}$ on $\left(m_{\sigma} / m_{\tilde{a}}, m_{X} / m_{\sigma}\right)$ plane. Left (right) panel corresponds to the KSVZ (DFSZ) model. We take $m_{\tilde{a}}=20 \mathrm{GeV}, f_{a}=10^{11} \mathrm{GeV}, \lambda_{332}^{\prime \prime}=$ $\left(3 \times 10^{6} \mathrm{GeV} / m_{X}\right)^{1 / 4} / 2$ and $\delta X_{\mathrm{ini}}=\delta \sigma_{\mathrm{ini}}=M_{\mathrm{Pl}}$ for both models, and $\mu=10^{2} m_{\tilde{a}}$ for the DFSZ model. In the plot shaded regions are excluded. "BBN" region is excluded due to $T_{X}<10 \mathrm{MeV}$ and "overabundant" means the region where $\Omega_{\tilde{a}}>\Omega_{\mathrm{DM}}$. The others are described in the figure. $m_{X}=3 \times 10^{6} \mathrm{GeV}$ is drawn in (blue) dash-dotted line (also indicated as "baryogenesis") to show that successful baryogenesis is realized in region above the line. $\Delta N_{\text {eff }}<1$ is satisfied in the region below the line $\Delta N_{\mathrm{eff}}=1$. For reference, contour of $\tau_{\tilde{a}}=10^{27} \mathrm{sec}$ is also plotted in (green) dotted line.

eq. (2.32). In the DFSZ model, axino is copiously produced when $T_{X}$ become larger than $\mu$, which soon becomes overabundant. Therefore, the line $\Omega_{\tilde{a}}^{\mathrm{TH}}=\Omega_{\mathrm{DM}}$ locates near $T_{X} \sim \mu$. Regarding axion dark radiation, we have found that $\Delta N_{\text {eff }}$ is less than unity in the valid parameter region. To be concrete, the constraint $\Delta N_{\text {eff }}<1$ is always satisfied in the region $\Gamma_{\sigma}>\Gamma_{X}$, independent of the parameters. (See eq. (2.35).)

Here we have a comment on the constraint due to overabundant axino from saxion decay. This constraint depends on the initial amplitude of the saxion after inflation as mentioned (see eqs. (2.11) and (2.31)). If we take smaller value for $\delta \sigma_{\text {ini }}$, such as $O\left(f_{a}\right)$, then the constraint becomes less stringent and smaller $m_{\sigma} / m_{\tilde{a}}$ region is allowed (e.g., if $\delta \sigma_{\text {ini }} \sim 10^{12} \mathrm{GeV}$, the constraint is irrelevant in the parameter space shown in the figure, which is easily read from eq. (2.31)). Such a mass spectrum might be realized in axionic mirage mediation or mixed SUSY breaking with anomalous U(1). [15, 66, 67]. The mass spectrum and the abundances of axino and axion strongly depend on the detail of the SUSY breaking mechanism. Further analysis will be given elsewhere in the future publication.

We found the upper bound for axino mass. For large axino mass, the bound from the lifetime becomes stringent. In order to suppress the decay width of axino, large soft mass (i.e. moduli mass) is needed. In the KSVZ model, however, large axino mass and the soft mass enhance the thermal production of axino (see eq. (2.32)). Then we found numerically

$$
m_{\tilde{a}} \lesssim 1 \times 10^{2} \mathrm{GeV}
$$


by taking $f_{a}=10^{15} \mathrm{GeV}$. This bound can be also read from figure 1 . When axino mass is larger than $O(100 \mathrm{GeV})$, axino decays to $W b b s$ where $W$ boson is on-shell, which leads to enhance the decay rate. As a consequence, the constraint from the lifetime and the overabundant bound destroy viable parameter region. In the DFSZ model, the bound from the lifetime becomes stringent for large axino mass as well. In this case the thermal production after moduli decay can be avoided if large $\mu$ is taken. However, the production during moduli domination is enhanced instead for large $\mu$, which leads to overabundant axino. Then we found numerically the upper bound for axino mass as

$$
m_{\tilde{a}} \lesssim 10^{5} \mathrm{GeV}
$$

while taking $f_{a}=10^{15} \mathrm{GeV}$.

There is no lower bound for axino mass in this context. Then it is possible to consider very large moduli mass. Let us suppose that moduli mass is $O\left(10^{16} \mathrm{GeV}\right) .\left(m_{X} \gtrsim 10^{16} \mathrm{GeV}\right.$ is invalid since $T_{X}$ may be as large as the soft mass scale, which may erase the baryon asymmetry.) With such a large $m_{X}$ and small $m_{\tilde{a}}$, axino relic is mainly from thermal production. Then axino with a mass of $O(10 \mathrm{keV})$ can be DM in the KSVZ model. In the DFSZ model, axino DM should have a mass of $m_{\tilde{a}} \sim O(0.1 \mathrm{MeV})$ when $f_{a}=10^{16} \mathrm{GeV}$ and $\mu \sim 10^{14} \mathrm{GeV}$, for example. If the BICEP2 result is confirmed, moduli mass should be larger than around $10^{16} \mathrm{GeV}$ in order for moduli to be stabilized. ${ }^{20}$ Therefore our scenario is parametrically compatible with high-scale inflation while stabilizing moduli although we need further analysis to get such a hierarchical mass spectrum.

Finally we discuss the experimental signatures involved in the scenario. Near the region $\tau_{\tilde{a}} \sim 10^{26-27} \mathrm{sec}$, the decay of axino produces hadrons and leptons, which may be observed as cosmic rays. Among them hadronic decay products are especially constrained by cosmicray anti-proton observation by PAMELA [69]. ${ }^{21}$ When axino mass is larger than order of a hundred $\mathrm{GeV}$, a large amount of high energy cosmic-ray anti-protons are generated. Then the cosmic rays will be detected by the AMS-02 experiment as an exotic signal, otherwise more stringent constraint will be given. If axino mass is smaller, the energy of the produced anti-proton gets smaller. In such a low energy range, the background cosmic ray increases. Thus it would be more difficult to see the signal, depending on the lifetime. If axino is lighter than $1 \mathrm{GeV}$, then axino becomes stable because it can not decay to the SM fermions. However, proton decays to axino via the RPV instead. As pointed out in ref. [73], $\lambda_{332}^{\prime \prime}$ induces $u d \tilde{s}$ type coupling, $\kappa_{u d s}$, which is $O\left(10^{-7}\right) \times$ $\lambda_{332}^{\prime \prime}$. Then proton decay to $K^{+} \tilde{a}$. The decay rate of proton is estimated as $\Gamma_{p \rightarrow K^{+} \tilde{a}} \sim$ $\frac{m_{p}}{16 \pi}\left(\frac{\tilde{\Lambda}_{\mathrm{QCD}}}{m_{\text {soft }}}\right)^{4}\left|\kappa_{u d s} g_{\mathrm{eff}}^{(L / R)}\right|^{2}$. Here $\tilde{\Lambda}_{\mathrm{QCD}} \sim 250 \mathrm{MeV}$ is the QCD scale. Then the lifetime of proton is estimated as $\tau_{p \rightarrow K^{+} \tilde{a}} \sim 3 \times 10^{32} \mathrm{yr}\left(\frac{f_{a}}{10^{10} \mathrm{GeV}}\right)^{2}\left(\frac{m_{X}}{10^{10} \mathrm{GeV}}\right)^{4}\left(\frac{4}{\log \left(f_{a} / m_{X}\right)}\right)^{2}\left(\frac{250 \mathrm{MeV}}{\tilde{\Lambda}_{\mathrm{QCD}}}\right)^{4}$ in the KSVZ model, and $\tau_{p \rightarrow K^{+} \tilde{a}} \sim 5 \times 10^{35} \mathrm{yr}\left(\frac{f_{a}}{10^{10} \mathrm{GeV}}\right)^{2}\left(\frac{m_{X}}{10^{8} \mathrm{GeV}}\right)^{4}\left(\frac{250 \mathrm{MeV}}{\tilde{\Lambda}_{\mathrm{QCD}}}\right)^{4}$ in DFSZ model. Here we have used $m_{\text {soft }}=m_{X} / 50$ and $\lambda_{332}^{\prime \prime} \sim 0.07$ and 0.2 in the KSVZ and the DFSZ models, respectively. The current experimental bound is $\tau_{p \rightarrow K^{+} \nu} \geq 2.3 \times 10^{33} \mathrm{yr}$ [74].

\footnotetext{
${ }^{20}$ See, e.g., a recent work [68], which takes into account the back-reaction effect.

${ }^{21}$ See earlier works, e.g., [70-72], which study cosmic-ray anti-proton from decaying DM.
} 
Therefore, proton decay experiment in the future could be a test of this scenario even in high moduli mass (soft mass) region.

In our scenario, lighter neutral Higgsino may be the LSP in the MSSM sector and as light as $O(100 \mathrm{GeV}-1 \mathrm{TeV})$. If the Higgsino is produced at a collider, it would decay inside the detector via the $O(1)$ RPV. However, its decay width is suppressed by the soft mass, thus it would decay from the interaction point. Even if the decay width of Higgsino is so suppressed by the soft mass that the decay occurs far from the interaction point, the decay would be observed. By counting the number of the decay events, the lifetime might be determined [75]. Then it may be possible to probe the validity of this scenario in the high soft mass region.

\section{Conclusion}

In this paper we consider axino dark matter in large $R$-parity violation. While moduli dominate the universe after inflation, saxion also oscillates coherently and eventually decays to produce large amount of LSP axino. Axino is also produced thermally at the reheating after inflation or lower temperature, depending on axion model. Such axinos are diluted by late moduli decay. We have found that the axino relic can give the correct amount to explain the present dark matter abundance in both the KSVZ and the DFSZ models. Though axino is metastable due to the large $R$-parity violation, its decay rate is suppressed the axion decay constant, the soft SUSY breaking mass or kinematics. Then the lifetime can be longer in order axino not to produce exotic cosmic rays. With the large $R$-parity violation, baryon asymmetry is generated in moduli-induced baryogenesis as well. Therefore the scenario explains both dark matter and baryon existing in the present universe.

\section{Acknowledgments}

We are grateful to Wilfried Buchmüller for discussions and giving fruitful comments and suggestions during the research. We are also thankful to Kwang Sik Jeong, Fuminobu Takahashi and Martin Winkler for useful discussions.

Open Access. This article is distributed under the terms of the Creative Commons Attribution License (CC-BY 4.0), which permits any use, distribution and reproduction in any medium, provided the original author(s) and source are credited.

\section{References}

[1] ATLAS collaboration, Observation of a new particle in the search for the Standard Model Higgs boson with the ATLAS detector at the LHC, Phys. Lett. B 716 (2012) 1 [arXiv: 1207.7214] [INSPIRE].

[2] CMS collaboration, Observation of a new boson at a mass of $125 \mathrm{GeV}$ with the CMS experiment at the LHC, Phys. Lett. B 716 (2012) 30 [arXiv:1207.7235] [INSPIRE].

[3] BICEP2 collaboration, P.A.R. Ade et al., Detection of B-mode polarization at degree angular scales by BICEP2, Phys. Rev. Lett. 112 (2014) 241101 [arXiv:1403.3985] [InSPIRE]. 
[4] R. Kallosh and A.D. Linde, Landscape, the scale of SUSY breaking and inflation, JHEP 12 (2004) 004 [hep-th/0411011] [INSPIRE].

[5] J.J. Blanco-Pillado, R. Kallosh and A.D. Linde, Supersymmetry and stability of flux vacua, JHEP 05 (2006) 053 [hep-th/0511042] [INSPIRE].

[6] M. Fukugita and T. Yanagida, Baryogenesis without grand unification, Phys. Lett. B 174 (1986) 45 [INSPIRE].

[7] I. Affleck and M. Dine, A new mechanism for baryogenesis, Nucl. Phys. B 249 (1985) 361 [INSPIRE].

[8] M. Dine, L. Randall and S.D. Thomas, Baryogenesis from flat directions of the supersymmetric Standard Model, Nucl. Phys. B 458 (1996) 291 [hep-ph/9507453] [InSPIRE].

[9] J.M. Cline and S. Raby, Gravitino induced baryogenesis: a problem made a virtue, Phys. Rev. D 43 (1991) 1781 [INSPIRE].

[10] S. Mollerach and E. Roulet, Axino induced baryogenesis, Phys. Lett. B 281 (1992) 303 [INSPIRE].

[11] K. Ishiwata, K.S. Jeong and F. Takahashi, Moduli-induced baryogenesis, JHEP 02 (2014) 062 [arXiv:1312.0954] [INSPIRE].

[12] S. Kachru, R. Kallosh, A.D. Linde and S.P. Trivedi, De Sitter vacua in string theory, Phys. Rev. D 68 (2003) 046005 [hep-th/0301240] [INSPIRE].

[13] R.D. Peccei and H.R. Quinn, CP conservation in the presence of instantons, Phys. Rev. Lett. 38 (1977) 1440 [INSPIRE].

[14] R.D. Peccei and H.R. Quinn, Constraints imposed by CP conservation in the presence of instantons, Phys. Rev. D 16 (1977) 1791 [INSPIRE].

[15] S. Nakamura, K.-I. Okumura and M. Yamaguchi, Axionic mirage mediation, Phys. Rev. D 77 (2008) 115027 [arXiv:0803.3725] [InSPIRE].

[16] H. Baer, K.-Y. Choi, J.E. Kim and L. Roszkowski, Non-thermal dark matter: supersymmetric axions and other candidates, arXiv:1407.0017 [INSPIRE].

[17] T. Goto and M. Yamaguchi, Is axino dark matter possible in supergravity?, Phys. Lett. B 276 (1992) 103 [InSPIRE].

[18] E.J. Chun and A. Lukas, Axino mass in supergravity models, Phys. Lett. B 357 (1995) 43 [hep-ph/9503233] [INSPIRE].

[19] E.J. Chun, J.E. Kim and H.P. Nilles, Axino mass, Phys. Lett. B 287 (1992) 123 [hep-ph/9205229] [INSPIRE].

[20] J.E. Kim and M.-S. Seo, Mixing of axino and goldstino and axino mass, Nucl. Phys. B 864 (2012) 296 [arXiv:1204.5495] [INSPIRE].

[21] M. Endo, K. Hamaguchi and F. Takahashi, Moduli-induced gravitino problem, Phys. Rev. Lett. 96 (2006) 211301 [hep-ph/0602061] [INSPIRE].

[22] M. Endo, K. Hamaguchi and F. Takahashi, Moduli/inflaton mixing with supersymmetry breaking field, Phys. Rev. D 74 (2006) 023531 [hep-ph/0605091] [INSPIRE].

[23] S. Nakamura and M. Yamaguchi, Gravitino production from heavy moduli decay and cosmological moduli problem revived, Phys. Lett. B 638 (2006) 389 [hep-ph/0602081] [INSPIRE]. 
[24] M. Dine, R. Kitano, A. Morisse and Y. Shirman, Moduli decays and gravitinos, Phys. Rev. D 73 (2006) 123518 [hep-ph/0604140] [INSPIRE].

[25] M. Kawasaki, N. Kitajima and K. Nakayama, Cosmological aspects of inflation in a supersymmetric axion model, Phys. Rev. D 83 (2011) 123521 [arXiv:1104.1262] [INSPIRE].

[26] T. Moroi and M. Takimoto, Thermal effects on saxion in supersymmetric model with Peccei-Quinn symmetry, Phys. Lett. B 718 (2012) 105 [arXiv:1207.4858] [INSPIRE].

[27] T. Moroi, K. Mukaida, K. Nakayama and M. Takimoto, Scalar trapping and saxion cosmology, JHEP 06 (2013) 040 [arXiv:1304.6597] [INSPIRE].

[28] J.E. Kim, Weak interaction singlet and strong CP invariance, Phys. Rev. Lett. 43 (1979) 103 [INSPIRE].

[29] M.A. Shifman, A.I. Vainshtein and V.I. Zakharov, Can confinement ensure natural CP invariance of strong interactions?, Nucl. Phys. B 166 (1980) 493 [INSPIRE].

[30] M. Dine, W. Fischler and M. Srednicki, A simple solution to the strong CP problem with a harmless axion, Phys. Lett. B 104 (1981) 199 [INSPIRE].

[31] A.R. Zhitnitsky, On possible suppression of the axion hadron interactions (in Russian), Sov. J. Nucl. Phys. 31 (1980) 260 [Yad. Fiz. 31 (1980) 497] [INSPIRE].

[32] L. Covi, J.E. Kim and L. Roszkowski, Axinos as cold dark matter, Phys. Rev. Lett. 82 (1999) 4180 [hep-ph/9905212] [INSPIRE].

[33] L. Covi, H.-B. Kim, J.E. Kim and L. Roszkowski, Axinos as dark matter, JHEP 05 (2001) 033 [hep-ph/0101009] [INSPIRE].

[34] K.-Y. Choi, L. Covi, J.E. Kim and L. Roszkowski, Axino cold dark matter revisited, JHEP 04 (2012) 106 [arXiv:1108.2282] [INSPIRE].

[35] A. Brandenburg and F.D. Steffen, Axino dark matter from thermal production, JCAP 08 (2004) 008 [hep-ph/0405158] [INSPIRE].

[36] A. Strumia, Thermal production of axino dark matter, JHEP 06 (2010) 036 [arXiv: 1003.5847] [INSPIRE].

[37] K. Rajagopal, M.S. Turner and F. Wilczek, Cosmological implications of axinos, Nucl. Phys. B 358 (1991) 447 [inSPIRE].

[38] E.J. Chun, Dark matter in the Kim-Nilles mechanism, Phys. Rev. D 84 (2011) 043509 [arXiv:1104.2219] [INSPIRE].

[39] K.J. Bae, K. Choi and S.H. Im, Effective interactions of axion supermultiplet and thermal production of axino dark matter, JHEP 08 (2011) 065 [arXiv:1106.2452] [INSPIRE].

[40] K.J. Bae, E.J. Chun and S.H. Im, Cosmology of the DFSZ axino, JCAP 03 (2012) 013 [arXiv:1111.5962] [INSPIRE].

[41] Planck collaboration, P.A.R. Ade et al., Planck 2013 results. XVI. Cosmological parameters, Astron. Astrophys. (2014) [arXiv:1303.5076] [INSPIRE].

[42] G. Mangano et al., Relic neutrino decoupling including flavor oscillations, Nucl. Phys. B 729 (2005) 221 [hep-ph/0506164] [INSPIRE].

[43] A.G. Riess et al., A 3\% solution: determination of the Hubble constant with the Hubble Space Telescope and Wide Field Camera 3, Astrophys. J. 730 (2011) 119 [Erratum ibid. 732 (2011) 129] [arXiv: 1103.2976] [INSPIRE]. 
[44] WMAP collaboration, G. Hinshaw et al., Nine-year Wilkinson Microwave Anisotropy Probe (WMAP) observations: cosmological parameter results, Astrophys. J. Suppl. 208 (2013) 19 [arXiv: 1212.5226] [INSPIRE].

[45] Atacama Cosmology Telescope collaboration, J.L. Sievers et al., The Atacama Cosmology Telescope: cosmological parameters from three seasons of data, JCAP 10 (2013) 060 [arXiv: 1301.0824] [INSPIRE].

[46] K.T. Story et al., A measurement of the Cosmic Microwave Background damping tail from the 2500-square-degree SPT-SZ survey, Astrophys. J. 779 (2013) 86 [arXiv:1210.7231] [INSPIRE].

[47] Z. Hou et al., Constraints on cosmology from the Cosmic Microwave Background power spectrum of the 2500-square degree SPT-SZ survey, Astrophys. J. 782 (2014) 74 [arXiv: 1212.6267] [INSPIRE].

[48] K.S. Jeong and F. Takahashi, Light higgsino from axion dark radiation, JHEP 08 (2012) 017 [arXiv:1201.4816] [INSPIRE].

[49] K. Choi, K.-Y. Choi and C.S. Shin, Dark radiation and small-scale structure problems with decaying particles, Phys. Rev. D 86 (2012) 083529 [arXiv: 1208.2496] [INSPIRE].

[50] M.S. Turner, Cosmic and local mass density of invisible axions, Phys. Rev. D 33 (1986) 889 [INSPIRE].

[51] M.P. Hertzberg, M. Tegmark and F. Wilczek, Axion cosmology and the energy scale of inflation, Phys. Rev. D 78 (2008) 083507 [arXiv:0807.1726] [INSPIRE].

[52] D.J.E. Marsh, D. Grin, R. Hlozek and P.G. Ferreira, Tensor detection severely constrains axion dark matter, Phys. Rev. Lett. 113 (2014) 011801 [arXiv:1403.4216] [INSPIRE].

[53] L. Visinelli and P. Gondolo, Axion cold dark matter in view of BICEP2 results, Phys. Rev. Lett. 113 (2014) 011802 [arXiv:1403.4594] [INSPIRE].

[54] T. Hiramatsu, M. Kawasaki, K. Saikawa and T. Sekiguchi, Production of dark matter axions from collapse of string-wall systems, Phys. Rev. D 85 (2012) 105020 [Erratum ibid. D 86 (2012) 089902] [arXiv: 1202.5851] [INSPIRE].

[55] S.M. Barr and J.E. Kim, New confining force solution of QCD domain wall problem, arXiv: 1407.4311 [INSPIRE].

[56] R. Flauger, J.C. Hill and D.N. Spergel, Toward an understanding of foreground emission in the BICEP2 region, JCAP 08 (2014) 039 [arXiv: 1405.7351] [INSPIRE].

[57] C. Cheung and K. Ishiwata, Baryogenesis with higher dimension operators, Phys. Rev. D 88 (2013) 017901 [arXiv: 1304.0468] [InSPIRE].

[58] R. Barbier et al., R-parity violating supersymmetry, Phys. Rept. 420 (2005) 1 [hep-ph/0406039] [INSPIRE].

[59] B. Bhattacherjee, J.L. Evans, M. Ibe, S. Matsumoto and T.T. Yanagida, Natural supersymmetry's last hope: R-parity violation via $U D D$ operators, Phys. Rev. D 87 (2013) 115002 [arXiv:1301.2336] [INSPIRE].

[60] I. Hinchliffe and T. Kaeding, $B+L$ violating couplings in the minimal supersymmetric Standard Model, Phys. Rev. D 47 (1993) 279 [InSPIRE].

[61] H.-B. Kim and J.E. Kim, Late decaying axino as CDM and its lifetime bound, Phys. Lett. B 527 (2002) 18 [hep-ph/0108101] [INSPIRE]. 
[62] D. Hooper and L.-T. Wang, Possible evidence for axino dark matter in the galactic bulge, Phys. Rev. D 70 (2004) 063506 [hep-ph/0402220] [InSPIRE].

[63] E.J. Chun and H.B. Kim, Axino light dark matter and neutrino masses with R-parity violation, JHEP 10 (2006) 082 [hep-ph/0607076] [INSPIRE].

[64] M. Endo, K. Hamaguchi, S.P. Liew, K. Mukaida and K. Nakayama, Axino dark matter with $R$-parity violation and $130 \mathrm{GeV}$ gamma-ray line, Phys. Lett. B 721 (2013) 111 [arXiv: 1301.7536] [INSPIRE].

[65] H. Murayama, I. Watanabe and K. Hagiwara, HELAS: HELicity amplitude subroutines for Feynman diagram evaluations, KEK-91-11, Japan (1992) [INSPIRE].

[66] K. Choi, K.S. Jeong, S. Nakamura, K.-I. Okumura and M. Yamaguchi, Sparticle masses in deflected mirage mediation, JHEP 04 (2009) 107 [arXiv: 0901.0052] [INSPIRE].

[67] K. Choi, K.S. Jeong, K.-I. Okumura and M. Yamaguchi, Mixed mediation of supersymmetry breaking with anomalous U(1) gauge symmetry, JHEP 06 (2011) 049 [arXiv:1104.3274] [INSPIRE].

[68] W. Buchmüller, C. Wieck and M.W. Winkler, Supersymmetric moduli stabilization and high-scale inflation, arXiv:1404.2275 [INSPIRE].

[69] PAMELA collaboration, O. Adriani et al., PAMELA results on the cosmic-ray antiproton flux from $60 \mathrm{MeV}$ to $180 \mathrm{GeV}$ in kinetic energy, Phys. Rev. Lett. 105 (2010) 121101 [arXiv: 1007.0821] [INSPIRE].

[70] A. Ibarra and D. Tran, Antimatter signatures of gravitino dark matter decay, JCAP 07 (2008) 002 [arXiv:0804.4596] [INSPIRE].

[71] K. Ishiwata, S. Matsumoto and T. Moroi, High energy cosmic rays from decaying supersymmetric dark matter, JHEP 05 (2009) 110 [arXiv:0903.0242] [INSPIRE].

[72] K. Ishiwata, S. Matsumoto and T. Moroi, Decaying dark matter in supersymmetric model and cosmic-ray observations, JHEP 12 (2010) 006 [arXiv: 1008.3636] [INSPIRE].

[73] S. Dimopoulos and L.J. Hall, Baryogenesis at the MeV era, Phys. Lett. B 196 (1987) 135 [INSPIRE].

[74] Super-Kamiokande collaboration, K. Kobayashi et al., Search for nucleon decay via modes favored by supersymmetric grand unification models in Super-Kamiokande-I, Phys. Rev. D 72 (2005) 052007 [hep-ex/0502026] [INSPIRE].

[75] K. Ishiwata, T. Ito and T. Moroi, Long-lived unstable superparticles at the LHC, Phys. Lett. B 669 (2008) 28 [arXiv:0807.0975] [INSPIRE]. 